\title{
On the Mixing Rate of the Triangulation Walk*
}

\author{
Michael S.O'B. MOLLOY \\ University of Toronto \\ molloy@cs.toronto.edu
}

\author{
BRUCE REED \\ CNRS - Paris, France \\ and IME - U. Sao Paulo, Brazil \\ reed@lug.ibp.fr
}

\author{
William Steiger \\ Rutgers University \\ steiger@cs.rutgers.edu
}

February 16, 1998

\begin{abstract}
Let $T_{n}$ denote the set of triangulations of a convex polygon $K$ with $n$ sides. We study the random walk on $T_{n}$ whose transitions are "flips" of one of the $n-3$ internal diagonals of the current triangulation, the choice of diagonal being random. By bounding the conductance of this graph we show that the walk mixes rapidly, namely in time $O\left(n^{\beta}\right)$. A direct argument is given for the fact that the mixing rate is at least $\Omega\left(n^{3 / 2}\right)$.
\end{abstract}

\section{Introduction and Summary}

Consider a convex polygon $K$ on $n$ points $p_{0}, \ldots, p_{n-1}$, in clockwise order. A triangulation is a set of $n-3$ non-crossing diagonals $p_{i} p_{j}$ which partitions $K$ into $n-2$ triangles. It is familiar (see e.g., [7]) that $T_{n}$, the set of all triangulations of $K$, satisfies

$$
\left|T_{n}\right|=\gamma_{n-2} \equiv \frac{1}{n-1}\left(\begin{array}{c}
2 n-4 \\
n-2
\end{array}\right)
$$

the right hand side being the $(n-2)^{n d}$ Catalan number. We consider the graph $G_{n}$ whose vertex set is $V=T_{n}$. Edges are triangulations which differ

\footnotetext{
${ }^{*}$ Part of the work of B. Reed was supported by a FAPESP grant
} 
by one diagonal. Specifically, a diagonal of a triangulation $\tau \in V$ may be "flipped"; i.e., removed - creating a quadrilateral - and replaced in the opposite way to give a new triangulation $\tau^{\prime}$ adjacent to $\tau$ in $G_{n}$. Thus $G_{n}$ is $n-3$ regular.

It is natural to consider the random walk on $G_{n}$ which moves to each neighbor of a vertex with equal chance. The key question is how quickly the walk converges to the steady-state distribution, in this case, the uniform. Often, there are practical reasons for studying such a random walk on a set $S$ of combinatorial objects, for example to estimate the size of $S$ or to generate an element of $S$ at random. However we already know $\left|G_{n}\right|=\gamma_{n-2}$ and there already exist optimal algorithms to generate random triangulations [3], [1], [2]. But there are interesting sets which are related to $T_{n}$, e.g., $T_{n}(P)$, the set of triangulations of the non-convex, simple polygon $P$, and $T_{n}(K)$, the triangulations of the set $K$ whose points are not in convex position; in both these cases the task of computing the size, and of generating a random element are open problems. By studying the walk on $T_{n}$ we may learn how to study other sets of triangulations.

Unfortunately little is known about the triangulation walk. This is surprising because $G_{n}$ is so familiar and well-studied [7], [12] ( also [4], [5], [6], [8], [10], which are relevant because $G_{n}$ is also the graph of rooted binary trees with $n-2$ internal nodes, adjacency defined by the rotation operation). In the present paper we will apply the method of conductance to show that the walk mixes rapidly. Write $\bar{S}$ for $T_{n} \backslash S$. We prove

Theorem 1 For any set $S \subset T_{n}$, there is a matching from $S$ to $\bar{S}$ with

$$
\geq \frac{|S||\bar{S}|}{\left|T_{n}\right| n^{\beta}}
$$

edges of $G_{n}$.

This would imply that the conductance is greater than $1 /\left(2 n^{\beta+1}\right)$ and the mixing rate $O\left(n^{2 \beta+3}\right)$ [11]. We will prove this with $\beta=11$ but think we can reduce it to 4 or 5 . In addition we show

Theorem 2 The mixing rate is at least $\Omega\left(n^{3 / 2}\right)$.

It is interesting here to compare this to the walk on the $n$-cube (similar to $G_{n}$, it is an $n$-regular graph with $c^{n}$ vertices), which mixes in $O(n \log n)$ steps. 
A key idea behind these results is a partitioning of $T_{n}$ into sets in which all triangulations share a certain "central triangle" $t$. This allows us to apply induction on the 3 subpolygons defined by $t$. It also gives us the ability to move between sets of triangulations in a systematic way and to construct a matching with many edges from $S$ to $\bar{S}$.

In the next section we collect some notations, definitions, and useful facts about $T_{n}$. The lower bound is proved in Section 3 . We do this first because it is much more pleasant and easier than the proof of the conductance bound, which is described in Section 4.

\section{Some Preliminaries}

Again, $G_{n}$ is the graph with vertex set $V=T_{n}$, the set of triangulations of the convex polygon $K$ whose vertices are the points $p_{0}, \ldots, p_{n-1}$, written in clockwise order; triangulations $\tau_{1}$ and $\tau_{2}$ in $V$ are adjacent if $\tau_{2}$ may be obtained by flipping one of the $n-3$ diagonals of $\tau_{1}$. The triangulation walk moves from a vertex $\tau$ to one of its $n-3$ neighbors, each with equal probability. This gives an ergodic Markov chain $M C(n)$ which is reversible, and its stationary distribution is the uniform probability on $V$.

If $p_{i} p_{j}$ is a diagonal of a triangulation $\tau \in V, i>j$, define its length to be $\left\|p_{i} p_{j}\right\|=\min (i-j, n-i+j)$, the (fewest) number of successive edges of $K$ between the endpoints. Every triangulation has a longest diagonal $\ell$ and it is clear that $n / 3 \leq\|\ell\| \leq n / 2$, the upper bound attainable only if $n$ is even. The longest diagonal must be in a triangle $t=\Delta p_{i} p_{j} p_{k}$, which has the sum of the lengths of its three diagonals equal to $n$. We call $t$ a central triangle and observe that it is unique unless $n$ is even and $\tau$ has a diagonal $\ell$ with length $n / 2$. In this case there are two such triangles, and to clarify the situation, we call $\ell=p_{i} p_{i+n / 2}$ a central edge and regard it as a degenerate central triangle. The diagonals comprising a nondegenerate central triangle $t=\Delta p_{i} p_{j} p_{k}$ divide $K$ into three subpolygons of sizes $\left\|p_{i} p_{j}\right\|+1,\left\|p_{j} p_{k}\right\|+1$, and $\left\|p_{k} p_{i}\right\|+1$. Write

$$
C_{n}=\left\{t=\Delta p_{i} p_{j} p_{k}: t \text { a central triangle }\right\}
$$

and observe that $\left|C_{n}\right|=\Theta\left(n^{3}\right)$. In fact $\left|C_{n}\right| \leq n^{3} / 12$ for $n \geq 10$.

It will be useful to regard central triangles as vertices in a graph, $G\left(C_{n}\right)$. A non-degenerate central triangle $t=\Delta p_{i} p_{j} p_{k}$ is adjacent to another nondegenerate central triangle $t^{\prime}$ if $t$ and $t^{\prime}$ have two points of $K$ in common and the third points differ by exactly one. A degenerate central triangle 
$\mathbf{K}$

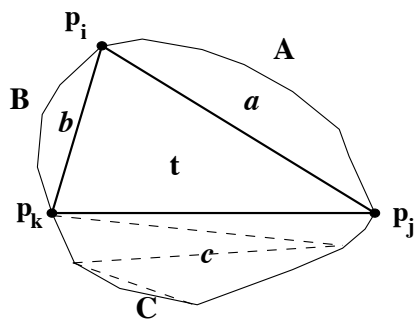

$\mathbf{V}=\mathbf{T}_{\mathbf{n}}$

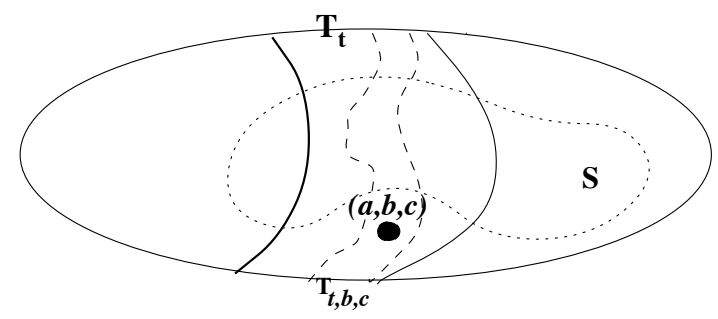

Figure 1: Triangulations with central triangle $t$. Subpolygon $C$ is triangulated by c. $\tau=(a, b, c)$ is in $\bar{S} \cap T_{t} . T_{t, b, c}$ denotes triangulations in $T_{t}$ with $b$ triangulating $B$ and $c$ triangulating $C$.

$p_{i} p_{i+n / 2}$ has four neighbors, $p_{i-1} p_{i-1+n / 2} ; p_{i+1} p_{i+1+n / 2}, \Delta p_{i-1} p_{i+1} p_{i+n / 2}$, and $\Delta p_{i} p_{i-1+n / 2} p_{i+1+n / 2}$. A central triangle has at most 6 neighbors in $G\left(C_{n}\right)$.

For each $t \in C_{n}$ we let $T_{t}$ denote the triangulations $\tau$ which have $t$ as their central triangle. The sets $T_{t}$ partition $V$. Given a central triangle $t=$ $\Delta p_{i} p_{j} p_{k}, i<j<k$, let $A$ denote the subpolygon $p_{i}, p_{i+1}, \ldots, p_{j}, p_{i}$ defined by $p_{i} p_{j}, B$, the subpolygon defined by $p_{i} p_{k}$, and $C$, the subpolygon defined by $p_{j} p_{i}$ (see Figure 1 ). We write $T_{A}, T_{B}$, and $T_{C}$ for the triangulations of $A$, $B$, and $C$, respectively. Observe that there are two degenerate cases here. The first is when $n$ is even and $t$ is degenerate. In this case we think of $A$ as a polygon with one vertex and $\left|T_{A}\right|=1$. In the other, $n$ is odd and $t$ contains an edge of $K$. In this case we think of $A$ as a polygon with two vertices and again $\left|T_{A}\right|=1$. We note that each triple $a \in T_{A}, b \in T_{B}, c \in T_{C}$ gives a distinct triangulation $\tau=(a, b, c) \in T_{t}$, and that

$$
T_{t}=\bigcup_{a} \bigcup_{b}(a, b, c)
$$

so $\left|T_{t}\right|=\left|T_{A}\right|\left|T_{B}\right|\left|T_{C}\right|$. In fact suppose that $\left\|p_{i} p_{j}\right\|=q,\left\|p_{j} p_{k}\right\|=r$, and that $\left\|p_{k} p_{i}\right\|=s$, where $p+q+r=n$ and $n / 3 \leq \max (q, r, s) \leq n / 2$. This means that $A$ has $q+1$ sides so (1) gives $\left|T_{A}\right|=\gamma_{q-1}$. Similarly $\left|T_{B}\right|=\gamma_{r-1}$ and $\left|T_{C}\right|=\gamma_{s-1}$, and we have

$$
\left|T_{t}\right|=\gamma_{q-1} \gamma_{r-1} \gamma_{s-1}
$$

This expression is maximized when $q=0$ and $r=s=n / 2$ (a degenerate 
central triangle) and minimized when $q=r=s=n / 3$. Since

$$
\gamma_{j} \sim \frac{4^{j}}{\sqrt{\pi j^{3}}}
$$

this implies

$$
\frac{9}{n^{3}} \leq \frac{\left|T_{t}\right|}{|V|} \leq \frac{5}{n^{3 / 2}}
$$

One consequence is that for $n \geq 20,\left|T_{t}\right| \leq|V| / 16$ for degenerate central triangles.

A diagonal $p_{i} p_{i+2}$ of length 2 defines an ear at $p_{i+1}$, and every $\tau \in V$ has at least 2 (and at most $n / 2$ ) ears. Define

$$
E_{i} \equiv\left\{\tau \in V: \tau \text { has an ear at } p_{i}\right\}, i=1, \ldots, n,
$$

the triangulations with ears at vertex $p_{i}$. Clearly $\left|E_{i}\right|=\left|T_{n-1}\right|$ so from (1),

$$
\left|E_{i}\right|=\frac{1}{4}\left|T_{n}\right|\left(1+\frac{3}{2 n-5}\right)
$$

The edge $p_{0} p_{1}$ of $K$ must be in some triangle of the triangulation, say $\Delta p_{0} p_{1} p_{i}$. According to (3), for about $1 / 4$ of the triangulations in $T_{n}, p_{i}=2$ (this puts an ear at $p_{1}$ ), and in about $1 / 4$ of the triangulations $p_{i}=n-1$ (an ear at $p_{0}$ ). If a triangulation $\tau$ contains triangle $t=\Delta p_{0} p_{1} p_{i}$ we write $A P E X_{n}(\tau)=\min (i, n-i), 2 \leq i \leq n-1$, the "distance" of the apex of $t$ from its base, $p_{0} p_{1}$. By (1), the number of triangulations with $A P E X=i$ is

$$
\gamma_{i-2} \gamma_{n-i-1}
$$

This implies that the average

$$
A=\frac{1}{\left|T_{n}\right|} \sum_{\tau \in T_{n}} A P E X_{n}(\tau)=O(\sqrt{n}) ;
$$

also the variance $V=\sum_{\tau \in T_{n}}\left(A-A P E X_{n}(\tau)\right)^{2}=O\left(n^{3 / 2}\right)$. By symmetry, in a random triangulation, the apex of the triangle containing polygon edge $p_{j} p_{j+1}$ is expected to have distance $O(\sqrt{n})$ from that edge.

Finally, given central triangles $t$ and $t^{\prime}$,

1. There is a path $P: t=u_{0}, u_{1}, \ldots, u_{k}=t^{\prime}$ in $G\left(C_{n}\right)$ with no interior $u_{i}$ degenerate, and

2. $\left|T_{u_{i}}\right|$ is minimized on one of the endpoints unless both $t$ and $t^{\prime}$ have a side of length $\leq 2$. In this case we can guarantee that $\min \left(\left|T_{u_{i}}\right|, u_{i} \in\right.$ $P) \geq\left|T_{t}\right| / 64$. 


\section{A Lower Bound on the Mixing Rate}

We prove that the mixing rate is at least $\Omega\left(n^{3 / 2}\right)$. Let $t=\Delta p_{1} p_{n / 2} p_{3 n / 4}$ be the initial central triangle and we start the chain at a random element $\tau \in T_{t}$. The walk continues while the central triangle avoids the interval $I$ from $p_{2 n / 10}$ to $p_{3 n / 10}$, an interval of length $n / 10$ (see Fig. 2). The important fact is that the subset $S \subset V$ whose central triangles have at least one vertex in $I$ satisfies

$$
P=\frac{|S|}{|V|} \geq c
$$

for some constant $c>0$. Let $N$ denote the number of steps of $M C(n)$ starting at $\tau$ - before the walk hits a triangulation in $S$. We will show that

Lemma $1 E(N) \geq \Omega\left(n^{3 / 2}\right)$.

From this it is easy to show that for any function $w(n) \uparrow \infty$,

$$
\operatorname{Prob}\left\{N<\frac{n^{3 / 2}}{w(n)}\right\} \rightarrow 0 .
$$

Since $S$ has probability $P>0$, the walk cannot be within $\varepsilon$ of the uniform distribution in $n^{3 / 2} / w(n)$ steps. With a little more effort we can establish stronger concentration for $N$ and eliminate $w(n)$.

Proof: The initial central triangle $t$ is on the left half of $K$. The edge $\beta=p_{1} p_{n / 2}$ is called the bounding edge: no vertex of $t$ is to the right of it. Furthermore, the part of the triangulation to the right of the bounding edge is random. $\beta$ is flipped with probability $1 /(n-3)$ so $\geq n$ steps of the chain are expected before this flip. At the moment $\beta$ is flipped, it is in a triangle that is part of a random triangulation of the right side of $K$, call it $\Delta p_{1} p_{i} p_{n / 2}$. If $i \in I$ the walk stops. Otherwise one of the subpolygons $p_{1}, \cdots, p_{i}, p_{1}$ or $p_{i}, \cdots, p_{n / 2}, p_{i}$ includes $I$ and the corresponding diagonal $\left(p_{1} p_{i}\right.$ or $\left.p_{i} p_{n / 2}\right)$ becomes the new bounding edge $\beta_{1}$. According to (4), we expect one of the endpoints of the bounding edge to move towards $I$ by at most $d \sqrt{n}$ vertices.

We apply the same argument inductively to the current bounding edge $\beta_{k}$. It will always be the case that (see Fig. 2)

1. The part of the current triangulation to the right of $\beta_{k}$ is random.

2. The central triangle of the current triangulation is to the left of $\beta_{k}$. 


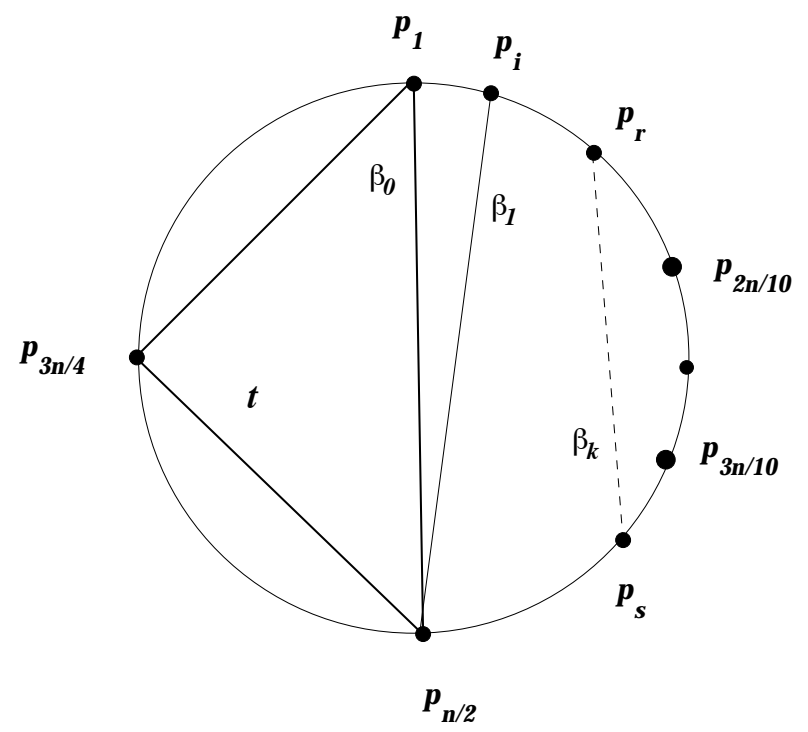

Figure 2: The Initial Central Triangle. $\beta_{0}=p_{1} p_{n / 2}$ is the initial bounding edge; $\beta_{k}$ is the edge after it has been flipped $k$ times.

3. At least $n$ steps of the chain are expected before $\beta_{k}$ is flipped.

4. If the walk continues, the new bounding edge $\beta_{k+1}$ has one if its vertices closer to $I$ than the corresponding vertex of $\beta_{k}$, and by an amount $\leq d \sqrt{n}$ vertices, expected.

Suppose the walk stops at the $M^{\text {th }}$ step of the chain. The previous facts imply that $E(M) \geq d^{-1} n^{3 / 2}$. Since no central triangle has yet had a vertex in $I, N \geq M$ and we are finished.

\section{The Conductance Bound}

The conductance $\Phi\left(G_{n}\right)$ is the minimum over all sets $S \subset V,|S| \leq|\bar{S}|$, of

$$
\frac{1}{n-3} \frac{\mid \text { edges } S \rightarrow \bar{S} \mid}{|S|} \text {. }
$$

Given $S \subset T_{n}$, write $r=|S| /\left|T_{n}\right|$ as its fraction of $V=T_{n}$. Theorem 1 asserts that for any $S \subset T_{n}$ there is a matching from $S$ to $\bar{S}$ with

$$
\geq \frac{|S||\bar{S}|}{\left|T_{n}\right| n^{\beta}}
$$


edges of $G_{n}$ and this implies that the conductance is greater than $1 /\left(2 n^{\beta+1}\right)$.

For a central triangle $t \in C_{n}$ write $S_{t}=S \cap T_{t}$ and $r_{t}=\left|S_{t}\right| /\left|T_{t}\right|$ as its fraction of $T_{t}$. To prove (5) we need the following facts.

Lemma 2 For any central triangle $t$ with $r_{t} \leq 12 / 23$, there is a matching with at least $\frac{2\left|S_{t}\right|}{n^{\beta}}$ edges from $S_{t}$ to $\bar{S} \cap T_{t}$.

Lemma 3 Consider central triangles $t_{1}$ with fraction $r_{1}=\left|S_{t_{1}}\right| /\left|T_{t_{1}}\right|$ and $t_{2}$ with $r_{2}=\left|S_{t_{2}}\right| /\left|T_{t_{2}}\right|$, and a path $P$ (respectively cycle $C$ ) containing them in $G\left(C_{n}\right)$. Let $M I N$ be the minimum of $\left|T_{t}\right|$ over all $t$ on $P$ (resp $C$ ). Then if no triangle internal to $P$ (resp. on $C$ ) is degenerate, there is a matching between $S \cap\left(\cup_{t \in P(\text { resp } C)} T_{t}\right)$ and $\bar{S} \cap\left(\cup_{t \in P(\text { resp } C)} T_{t}\right)$ with

$$
\geq \frac{31\left|r_{1}-r_{2}\right| M I N}{n^{8}}\left(\operatorname{resp} \frac{40\left|r_{1}-r_{2}\right| M I N}{n^{8}}\right)
$$

edges. If there is a degenerate triangle interior to $P$ (resp. on $C$ ) then we can find a matching with at least

$$
\geq \frac{7\left|r_{1}-r_{2}\right| M I N}{n^{8}}\left(\operatorname{resp} \frac{8\left|r_{1}-r_{2}\right| M I N}{n^{8}}\right)
$$

edges.

Lemma 4 Consider adjacent triangles $t_{1}$ and $t_{2}$ in $G\left(C_{n}\right)$. Write $r_{i}=$ $\left|S \cap T_{t_{i}}\right| /\left|T_{t_{i}}\right|$. If one of $t_{1}$ or $t_{2}$ is not degenerate then there is a matching between $S \cap\left(T_{t_{1}} \cup T_{t_{2}}\right)$ and $\bar{S} \cap\left(T_{t_{1}} \cup T_{t_{2}}\right)$ with

$$
\frac{63\left|r_{1}-r_{2}\right| \min \left(\left|T_{t_{1}}\right|,\left|T_{t_{2}}\right|\right)}{n^{8}}
$$

edges. If both $t_{1}$ and $t_{2}$ are degenerate then there is such a matching with at least

$$
\frac{15\left|r_{1}-r_{2}\right| \min \left(\left|T_{t_{1}}\right|,\left|T_{t_{2}}\right|\right)}{n^{8}}
$$

edges.

Proof of Theorem 1: We prove (5) with $\beta=11$. Because $r=|S| /\left|T_{n}\right| \leq$ $1 / 2$, the asserted size of the matching is less than 1 unless $\gamma_{n-2}>2 n^{11}$, so we proceed by induction on $n$, now assuming that $n \geq 35$. 
If there were big matchings from $S \cap T_{t}$ to $\bar{S} \cap T_{t}$ for enough of the $T_{t}$ we would be done. Let Big $=\left\{t \in C_{n}: r_{t}>12 / 23\right\}$ and $T_{B i g}=\bigcup_{t \in B i g} T_{t}$. Applying Lemma 2 (the proof is based on a straightforward induction argument (which is deferred to an appendix) to central triangles not in Big and summing, we find there is a matching of size

$$
2 \sum_{t \notin B i g} \frac{\left|S \cap T_{t}\right|}{n^{\beta}}=2 \frac{\left|S \cap \overline{T_{B i g}}\right|}{n^{\beta}} .
$$

The right hand side exceeds the asserted bound unless $\left|S \cap \overline{T_{B i g}}\right|<|S| / 2$, so we may assume $\left|S \cap T_{B i g}\right| \geq|S| / 2$.

This implies that there is a central triangle $t_{1}$ for which $r_{t_{1}} \geq 12 / 23$. Also, since $r \leq 1 / 2$, there is a $t_{2} \in C_{n}$ with $r_{t_{2}} \leq 1 / 2$ and consequently we have a pair of central triangles with $\left|r_{t_{1}}-r_{t_{2}}\right| \geq 1 / 46$. We apply the second bound from Lemma 3 . By (2), MIN $\geq 9|V| /\left(n^{3}\right)$, so there is a matching with at least

$$
\frac{63\left|r_{t_{1}}-r_{t_{2}}\right||V|}{n^{11}}
$$

edges. This says there is a matching with more than $63|S| /\left(23 n^{11}\right)$ edges.

Proof of Lemma 3: Take a 2 colouring of the edges of the path (respectively three colouring of the cycle). Choose the colour class which maximizes $\sum_{t_{a} t_{b} \in \text { colour class }}\left|r_{a}-r_{b}\right|$. To get the desired result, sum over the edges of this colour class, applying Lemma 4 to each edge.

Remark 1: Write $t_{1}=\Delta p_{i} p_{j} p_{k}$ and $t_{2}=p_{i} p_{j} p_{k^{\prime}},\left|k-k^{\prime}\right|=1$. If there were a big matching linking $T_{t_{1}}$ to $T_{t_{2}}$ then Lemma 4 would be true even without the $\frac{63}{n^{8}}$ factor. Unfortunately this never occurs; $T_{t_{1}}$ and $T_{t_{2}}$ are rarely even the same size. However there is a matching linking the subset $Y_{1}$ of $T_{t_{1}}$ in which $p_{k^{\prime}}$ is an ear in the quotient polygon containing $p_{k}$ with the subset $Y_{2}$ of $T_{t_{2}}$ in which $p_{k}$ is an ear in the quotient polygon containing $p_{k^{\prime}}$. In fact the edges between these two sets form a matching. Furthermore, $Y_{1}$ contains about a quarter of $T_{t_{1}}$ and $Y_{2}$ contains about a quarter of $T_{t_{2}}$. So, if $\frac{\left|S \cap Y_{i}\right|}{\left|Y_{i}\right|}$ were equal to $r_{i}$ then Lemma 4 would be true with $63 / n^{8}$, replaced by $\frac{1}{4}$. The only dificulty in the proof occurs when either $r_{1}$ is significantly different from $\frac{\left|S \cap Y_{1}\right|}{\left|Y_{1}\right|}$ or $r_{2}$ is significantly different from $\frac{\left|S \cap Y_{2}\right|}{\left|Y_{2}\right|}$.

To handle this situation, we apply the following lemma, which may have independent interest, to the two appropriate quotient polygons: 
Fix $m$ and write $S(m)=S \cap E_{m}$ for the elements of $S$ with ears at $p_{m}$, To estimate the number of edges entering $S(m)$, define the dispersion

$$
D_{m}(S)=|| S(m)|-| S\left|\frac{\left|E_{m}\right|}{\left|T_{n}\right|}\right| .
$$

This measures the deviation from the expected number of elements of $S$ with ears at $p_{m}$, assuming $S$ were a randomly chosen set of size $|S|$.

Lemma 5 (The "Ear Lemma") For any $m=1, \ldots, n$ there is a matching from $S$ to $\bar{S}$ with at least $D_{m}(S) / n^{8}$ edges of $G$.

Proof: We recursively prove Lemmas 4 and 5 in the following way: We prove Lemma 4 for any $n$ assuming Lemmas 4 and 5 for all $n^{\prime}<n$. We prove Lemma 5 for any $n$ assuming both lemmas for $n^{\prime}<n$ and Lemma 4 for $n$. The proofs proceed by induction on central triangles. Here is a sketch.

We can take $|S(m)| \geq|S|\left|E_{m}\right| /\left|T_{n}\right|$ in (6) since $D_{m}(S)=D_{m}(\bar{S})$ and the absolute value is redundant in one of these two cases. Given $t=\Delta p_{i} p_{j} p_{k} \in$ $C_{n}$, the ring $R=R(t)$ is the set

$$
\left\{t, \Delta p_{i+1} p_{j+1} p_{k+1}, \ldots, \Delta p_{i-1} p_{j-1} p_{k-1}\right\}
$$

of rotations of $t$ (subscripts always $\bmod (n))$. Write

$$
T_{R}=\bigcup_{t \in R} T_{t}
$$

and $S_{R}=S \cap T_{R}$, and let $\mathcal{R}$ be the set of all rings. A ring is non-degenerate if it consists of non-degenerate triangles.

For each $\ell=1, \ldots,\lfloor n / 3+1\rfloor$ the level $X_{\ell}$ is the set of $t \in C_{n}$ such that the minimum length quotient polygon has size $\ell$, and we write

$$
T_{X_{\ell}}=\bigcup_{t \in X_{\ell}} T_{t}
$$

and $S_{X_{\ell}}=S \cap T_{X_{\ell}}$. We note that $X_{1}$ is empty if $\ell$ is odd and $X_{2}$ is empty if $\ell$ is even. We need to treat $X_{1}$ and $X_{2}$ differently from $X_{\ell}, \ell \geq 3$, and we call then degenerate levels. Finally write for each $t \in C_{n}$, ring $R$, and $\ell$

$$
r=\frac{|S|}{\left|T_{n}\right|}, \quad r_{t}=\frac{\left|S_{t}\right|}{\left|T_{t}\right|}, \quad r_{R}=\frac{\left|S_{R}\right|}{\left|T_{R}\right|}, \quad r_{\ell}=\frac{\left|S_{X_{\ell}}\right|}{\left|T_{X_{\ell}}\right|},
$$


and observe that

$$
D_{m}(S)=\sum_{t \in C_{n}}\left(\left|S_{t} \cap S(m)\right|-\frac{|S|}{\left|T_{n}\right|}\left|T_{t} \cap E_{m}\right|\right) .
$$

If $p_{m}$ is a vertex of $t=\Delta p_{i} p_{j} p_{k}$ then $\left|T_{t} \cap E_{m}\right|=0$. Otherwise, letting $\lambda_{t}$ denote the size of the quotient subpolygon containing $p_{m}$ and using (3), it is $\left|T_{t}\right|\left(1+3 /\left(2 \lambda_{t}-5\right)\right) / 4$, and we have

$$
D_{m}(S)=\sum_{t \in C_{n}}\left(\left|S_{t} \cap S(m)\right|-\frac{r\left|T_{t}\right|}{4}\left(1+\frac{3}{2 \lambda_{t}-5}\right)\right),
$$

where we understand that $\lambda_{t}=1$ if $t$ contains $p_{m}$. Clearly

$$
\begin{gathered}
D_{m}(S)=\sum_{t \in C_{n}}\left(\left|S_{t} \cap S(m)\right|-\frac{r_{t}\left|T_{t}\right|}{4}\left(1+\frac{3}{2 \lambda_{t}-5}\right)\right) \\
+\sum_{\ell=1}^{n / 3} \sum_{R \in X_{\ell}} \sum_{t \in R}\left[\left(r_{t}-r_{R}\right)+\left(r_{R}-r_{\ell}\right)+\left(r_{\ell}-r\right)\right] \frac{1}{4}\left|T_{t}\right|\left(1+\frac{3}{2 \lambda_{t}-5}\right),
\end{gathered}
$$

and rewriting the second summations,

$$
\begin{gathered}
D_{m}(S)=\sum_{t \in C_{n}}\left(\left|S_{t} \cap S(m)\right|-\frac{r_{t}\left|T_{t}\right|}{4}\left(1+\frac{3}{2 \lambda_{t}-5}\right)\right) \\
+\frac{1}{4} \sum_{R \in \mathcal{R}} \sum_{t \in R}\left(r_{t}-r_{R}\right)\left|T_{t}\right|\left(1+\frac{3}{2 \lambda_{t}-5}\right) \\
+\frac{1}{4} \sum_{\ell=1}^{n / 3} \sum_{R \in X_{\ell}} \sum_{t \in R}\left(r_{R}-r_{\ell}\right)\left|T_{t}\right|\left(1+\frac{3}{2 \lambda_{t}-5}\right)+\frac{1}{4} \sum_{\ell=1}^{n / 3} \sum_{R \in X_{\ell}} \sum_{t \in R}\left(r_{\ell}-r\right)\left|T_{t}\right|\left(1+\frac{3}{2 \lambda_{t}-5}\right) .
\end{gathered}
$$

We deal with these four summation terms one at a time. The idea is that if any are large, then the lemma is already true. On the other hand they cannot all be small.

Let $\sigma_{1}$ be the value of the first summation in (8) and we write

$$
\sigma_{1}=\sum_{t \in C_{n}} D^{t}(S)
$$

where

$$
D^{t}(S)=\left|S_{t} \cap S(m)\right|-\frac{r_{t}\left|T_{t}\right|}{4}\left(1+\frac{3}{2 \lambda_{t}-5}\right) .
$$


We can interpret $D^{t}(S)$ as the dispersion in the quotient subpolygon containing $p_{m}$. Using Lemma 5 inductively and noting that the subpolygon has size $\lambda_{t}+1 \leq n / 2, D^{t}(S)$ accounts for at least $D^{t}(S) /(n / 2)^{8}$ edges from $S_{t}$ to $\bar{S} \cap T_{t}$. Summing over $C_{n}$ reveals that $D_{m}(S)$ accounts for at least $2^{8} \sigma_{1} / n^{8}$ edges from $S$ to $\bar{S}$, so unless $\sigma_{1} \leq D_{m}(S) / 2^{8}$, the ear lemma is already true.

Next consider

$$
\sigma_{2}=\frac{1}{4} \sum_{R \in \mathcal{R}} \sum_{t \in R}\left(r_{t}-r_{R}\right)\left|T_{t}\right|\left(1+\frac{3}{2 \lambda_{t}-5}\right),
$$

the second summation term in (8), and consider a non-degenerate ring $R$. It consists of rotations of a triangle $t$ with sides of length $a \leq b \leq c, a+b+c=n$, $n / 3 \leq c \leq n / 2$. For each $t$ in $R$ either (i) $p_{m}$ is in $t$ or $p_{m}$ is in the quotient polygon of size (ii) $a+1$, (iii) $b+1$, or (iv) $c+1$. For each ring $R$, the term in $\sigma_{2}$ is

$$
\begin{gathered}
\sum_{t \in R}\left(r_{t}-r_{R}\right) \frac{\left|T_{t}\right|}{4}\left(1+\frac{3}{2 \lambda_{t}-5}\right)=\sum_{t \in R} r_{t} \frac{\left|T_{t}\right|}{4}-r_{R} \sum_{t \in R} \frac{\left|T_{t}\right|}{4}+\sum_{t \in R} \frac{3}{2 \lambda_{t}-5} \frac{\left|T_{t}\right|}{4}\left(r_{t}-r_{R}\right) \\
=\frac{\left|S_{R}\right|}{4}-\frac{\left|S_{R}\right|}{4}+\sum_{t \in R} \frac{3}{2 \lambda_{t}-5} \frac{\left|T_{t}\right|}{4}\left(r_{t}-r_{R}\right) .
\end{gathered}
$$

Now, for each non-degenerate ring $R$, we let

$$
D_{R}(S)=\frac{1}{4} \sum_{t \in R} \frac{3}{2 \lambda_{t}-5}\left|T_{t}\right|\left(r_{t}-r_{R}\right)
$$

be the term in the $\sigma_{2}$ sum which corresponds to $R$. For each non-degenerate $R$, we can find a cycle $C_{R}$ in $G\left(C_{n}\right)$ containing no degenerate triangles, which contains all of $R$, and has exactly $3|R|$ vertices all of which are either in $R$ or adjacent to an element of $R$. We call $C_{R}$, the extended ring for $R$. Each central triangle is in at most 7 extended rings since it has at most six neighbours in $G\left(C_{n}\right)$. Let $T_{C_{R}}=\bigcup_{t \in C_{R}} T_{t}$.

Claim 1 (i) For each non-degenerate ring $R$, there is a matching between $S \cap T_{C_{R}}$ and $\bar{S} \cap T_{C_{R}}$ with at least $\frac{21}{20} D_{R}(S)$ edges. (ii) For the (at most one) degenerate ring $R$ that there is a matching from $S \cap T_{R}$ to $\bar{S} \cap T_{R}$ with at least $\sum_{t \in R} \frac{3}{2 \lambda_{t}-5}\left|T_{t}\right|\left(r_{t}-r_{R}\right)$ edges.

This claim is proved by choosing $t_{1}$ and $t_{2}$ which respectively maximize and minimize $r_{t}$ over $C_{R}$ and then applying Lemma 3 to $t_{1}, t_{2}$, and $C_{R}$. We omit 
the details. Since each central triangle is in at most seven extended rings, the union of these matchings over the non-degenerate triangles has maximum degree seven, and hence has an eight colouring. Adding the disjoint matching from the degenerate ring to the largest color class, and summing the contributions of all the rings, we see that if $\sigma_{2}$ accounts for more than $1 / 2$ of $D_{m}(S)$, Lemma 5 is already true.

Next we can apply Lemma 3 in a similar but more complicated fashion to show that the third term in (8), $\sigma_{3}$, must be less than $D_{m}(S) / 400$, and finally, that $\sigma_{4}$, the fourth summation in (8), is less than $5 D_{m}(S) / 12$. Putting these statements together, the ear lemma is already true unless

$$
D_{m}(S) \leq \frac{D_{m}(S)}{2^{8}}+\frac{D_{m}(S)}{2}+\frac{D_{m}(S)}{400}+\frac{5 D_{m}(S)}{12}<D_{m}(S) .
$$

an impossibility that completes the proof.

Proof of Lemma 4: We apply the Ear Lemma inductively,assuming it is true for polygons with at most $n-1$ sides. Suppose first the two triangles are non-degenerate. Let $t_{1}, t_{2}, Y_{1}, Y_{2}$ be as in Remark 1 . Let $r_{i}^{\prime}=\left|S \cap Y_{i}\right| /\left|Y_{i}\right|$. If $\left|r_{1}^{\prime}-r_{2}^{\prime}\right| \geq 64\left|r_{1}-r_{2}\right| / n^{8}$, then the result holds by considering the matching linking $Y_{1}$ and $Y_{2}$. Otherwise, $\left|r_{1}-r_{1}^{\prime}\right|+\left|r_{2}-r_{2}^{\prime}\right| \geq\left(1-\frac{65}{n^{8}}\right)\left|r_{1}-r_{2}\right|$. Since $Y_{i}$ is just $E_{m}$ for some ear in one of the quotient polygons defined by $t_{i}$, the result holds by applying the Ear Lemma to these two quotient polygon (we need to sum over all pairs of triangulations of the other polygons but this is straightforward). We get a $2^{8}$ factor bonus because the quotient polygons are smaller. This explains the 63 in Lemma 4 which is not present in the Ear Lemma. If exactly one of the $t_{i}$ is degenerate, we proceed as above, but now the matching is between a subset of $T_{t_{1}}$ where we fix an ear in both quotient polygons. However the matching hits all of $T_{t_{2}}$ so $M I N=\left|T_{t_{2}}\right|$ and the proof technique works. If $t_{1}$ and $t_{2}$ are both degenerate, then the fact that we consider two ears - not one - accounts for the loss of a factor of 4 in the result. We omit the details.

\section{References}

[1] D. Arnold and M. Sleep. Uniform Random Number Generation of $n$ Balanced Parenthesis Strings. ACM Trans. on Prog. Languages and Systems 2, 122-128, 1980. 
[2] M. Atkinson and J. Sack. Generating Binary Trees at Random. Inf. Proc. Letters 41, 21-23, 1992.

[3] L. Devroye, P. Flajolet, F. Hurtado, M. Noy, and W. Steiger. Random Triangulations. manuscript, 1996.

[4] L. Devroye. A Note on the Height of Binary Search Trees. J.A.C.M. 33, 489-498, 1986.

[5] L. Devroye. Application of the Theory of Records in the Study of Random Trees. Acta Informatica 26, 123-130, 1988.

[6] P. Flajolet and A. Odlyzko. The Average Height of Binary Trees and Other Simple Trees. J. Comp. Syst. Sciences, 25, 171-213, 1982.

[7] F. Hurtado and M. Noy. The Graph of Triangulations of a Convex Polygon. ACM Symposium on Computational Geometry (to appear), 1996.

[8] H. Mahmoud. Evolution of Random Search Trees. John Wiley, 1992.

[9] A. Meir and J. Moon. On the Altitude of Nodes in Random Trees. Canad. J. Math. 30, 997-1015, 1978.

[10] B. Pittel. On Growing Random Binary Trees. J. Math. Anal. Appl. 103, 461-480, 1984.

[11] A. Sinclair. Algorithms for Random Generation and Counting: A Markov Chain Approach. Birkhauser, Boston, 1992

[12] D. Sleator, R. Tarjan, and W. Thurston. Rotation Distance, Triangulations, and Hyperbolic Geometry. J. Amer. Math. Soc. 1, 647-681, 1988. 